\title{
Mid term outcomes in Wellens syndrome
}

\author{
Anamaria Avram ${ }^{1,2}$, Valentin Chionce ${ }^{1,2}$, Catalina Liliana Andrei ${ }^{1,2}$, Crina Sinescu $^{1,2}$ \\ ${ }^{1 " C}$ Carol Davila" University of Medicine and Pharmacy, Bucharest, Romania \\ 2"Bagdasar-Arseni" Emergency Clinical Hospital, Bucharest, Romania
}

\begin{abstract}
Background. Wellens syndrome (WS) has been described as a clinical and electrocardiographic (ECG) complex that identifies a subset of patients with unstable angina (UA) at an impending risk of myocardial infarction (MI) and death in studies published almost 4 decades ago, before the wide use of cardiac biomarkers such as troponins. The mid and long term outcomes of patients with Wellens syndrome have never been compared with a contemporary cohort of patients with non-ST elevation acute coronary syndromes (NSTEACS).

Objectives. The primary endpoints of our study were the rate of cardiovascular rehospitalizations, the rate of ischaemic reccurences, the rate of subsequent or reccurent revascularization and the rate of mortality at six months from the index event.

Materials and methods. We performed a prospective analysis of 64 consecutive patients with WS who underwent coronary angiography and we compared them with an age and sex matched cohort of patients with NSTEACS who underwent coronary angiography. The study took place at Bagsadar-Arseni Emergency Clinical Hospital and included a total of 127 patients recruited within 2 years (from January 2018 until December 2019), who were followed for a period of 6 months.

Results. Within 6 months of follow-up, patients in the control group had a significantly higher rate of cardiovascular rehospitalizations $(41.9 \%$ vs. $21.9 \%, p=0.016)$, although the rate of ischaemic recurrences was similar between the 2 groups. Other interventional end-points, such as subsequent interventional revascularization, repeat interventional revascularization and repeat target vessel revascularization (TVR) were comparable between the 2 groups at 6 months follow-up. There was no significantly difference with respect to global mortality $(6.3 \%$ in WS group vs. $7.9 \%$ in the control group, $p=0.74)$.

Discussions. To our knowledge, this is the first prospective study with mid term follow up that compared a consecutive cohort of patients with WS who underwent coronary angiography with an age and sex matched cohort of patients with NSTEACS. Patients with WS had similar event rates with respect to ischaemic recurrences, subsequent or repeat interventional revascularization and repeat target vessel revascularization (TVR) and mortality rate at 6 months, although significantly more patients in WS group were considered at low risk.

Conclusions. Wellens sign is frequently overlooked in the emergency department and risk assessment based on risk scores is frequently misleading. Prompt recognition of subtle ECG ischaemic changes, such as WS, in patients with chest pain is crucial, as it reflects a large area of myocardium at risk and identifies a subgroup of patients who can benefit from early invasive management.
\end{abstract}

Keywords: acute coronary syndrome, Wellens syndrome, risk stratification, revascularization, mortality

\section{BACKGROUND}

Acute coronary syndromes (ACS) are classified into ST segment elevation myocardial infarction (STEMI) and non-ST segment elevation ACS (NSTEACS) according to the ECG criteria, with diagnostic, therapeutic and prognostic implications. After sudden cardiac death, STEMI is the most severe manifestation of ischaemic heart disease, but it has the double advantage that it is quickly and easily diagnosed based on the ECG, on one hand, and that there is general agreement of the recommendations of the scientific societes regarding the benefit of immediate reperfusion treatment, on the other hand [1]. NSTEACS represent a heterogeneous group regarding the ECG manifestations and treatment strategy, as the priority level for invasive approach depends essentialy upon risk stratification [2]. Among the ECG presentations of 
NSTEACS, there is a series of particular phenotypes reffered to as STEMI equivalents because they are strongly correlated to impending coronary occlusion and transmural myocardial infarction (MI) [3]. Wellens syndrome has been described as a clinical and ECG complex that identifies a subset of patients with UA at an impending risk of MI and death in studies published almost 4 decades ago $[4,5]$, before the wide use of cardiac biomarkers such as troponins.

The medium and long term outcomes of patients with Wellens syndrome have never been compared with a contemporary cohort of patients with non-ST elevation acute coronary syndromes (NSTEACS).

\section{OBJECTIVES}

The primary endpoints of our study were the rate of cardiovascular rehospitalizations, the rate of ischaemic reccurences, the rate of subsequent or reccurent revascularization and the rate of mortality at the end of the follow up period.

\section{MATERIALS AND METHODS}

We performed a prospective analysis of 64 consecutive patients with Wellens syndrome who underwent coronary angiography between January 2018 and December 2019 and we compared them with an age and sex matched cohort of patients with NSTEACS who underwent coronary angiography within the same period of time. Patients follow-up visits were at one month and at six months from the index event. Inclusion criteria were: chest pain or equivalents; ischemic changes on ECG (horizontal or down-sloping ST-segment depression $\geq 0.05 \mathrm{mV}$ or $+/$ - biphasic/ negative T-waves in two or more contiguous leads) or absence of ST-T changes on ECG; coronary angiography performed during the reference hospitalization. Wellens syndrome has been defined as: chest pain or equivalents; +/- biphasic or negative T-waves in two or more contiguous leads; normal myocardial necrosis enzymes. Exclusion criteria were: ST segment elevation on ECG; pathological Q waves in leads V2 sand V3; left ventricular hypertrophy; complete left bundle branch block; ventricular paced rhythm; alternative diagnoses such as: acute myocarditis, acute pericarditis, acute pulmonary embolism, hypertrophic cardiomyopathy, arrhythmogenic cardiomyopathy, central nervous system injury. We screened 483 patients, of whom 127 were included in the final analysis. The pres- ent study complied with the Declaration of Helsinki requirements and was approved by the institutional review board. The patients signed the informed consent form to participate in the study and the educational process.

\section{Demographic, clinical and noninvasive data}

Demographic data have been obtained from medical history and medical records. Clinical data at admission have been extracted from medical records in the emergency department.

Standard 12-lead electrocardiograms, as well as aditional leads at the discretion of the treating physician, were obtained using Schiller devices soon after presentation and throughout the hospital stay. The ECG tracings met the standard settings for voltage $(10 \mathrm{~mm} / \mathrm{mV})$ and paper speed $(25 \mathrm{~mm} / \mathrm{s})$.

High sensitive troponin I (hs-cTnI) was measured using the PATHFAST ${ }^{\mathrm{TM}}$ immunoanalyzer (LSI Medience Corporation, a subsidiary of Mitsubishi Chemical Holdings). The overall cut-off for the $99^{\text {th }}$ percentile value is $27.9 \mathrm{ng} / \mathrm{l}$. Routine laboratory data included complete blood count, coagulation, ESR and biochemistry.

Transthoracic echocardiography was performed using the Aloka Prosound Alpha 7 machine and the Aloka UST - 52105 cardiac ultrasound probe. Standard morphological and hemodynamic parameters have been obtained following the recommendations of the EACVI (European Association of Cardiovascular Imaging) guidelines.

\section{Risk assessment}

Risk assessment has been made using validated risk scores. We used in-hospital GRACE 1.0 to guide the invasive approach and GRACE 2.0 for in-hospital and 6 months mortality estimation.

\section{Invasive data}

Angiographic data have been obtained using Phillips Allura Xper FD 10/10 system. All patient underwent coronary angiography during hospital stay. The results were reviewed by two independent interventional cardiologists. The following data were recorded: coronary artery disease inventory using visual assessment or quantitative algorithms such as QCA (quantitative coronary angiography), culprit lesion, treatment decision, number and type of the implanted stents, completeness of revascularization.

\section{Prognostic data}

Patients follow-up visits were at one month and at six months. Clinical, electrocardiographic, bio- 
logical and echocardiographic data were recorded at both follow-up visits.

\section{Statistical analysis}

Statistical analysis was performed using SPSS software (version 22.0). Continuous variables were compared using two-tailed student's T test. Continuous paired variables were compared using paired T-test. Categorical variables (mainly binary) were compared using Chi-squared test. For variables not normally distributed or for those with small values we used nonparametric tests, such as Wilkoxon rank-sum test for independent continuous variables and Fisher's exact test for independent categorical variables.

\section{RESULTS}

The study population consisted of 127 patients with a mean age of 62.38 years, of whom 33\% were women. Baseline demographic, clinical and noninvasive data are summarized in Table 1.
Baseline cardiovascular risk profile was comparable between the 2 groups, except for a larger proportion of diabetes mellitus (DM) in the control group. There were no differences regarding relevant cardiovascular history. Chronic outpatient treatment was similar between the 2 groups, except for a higher proportion of patients on statins in WS group. The symptom onset to presentation time interval was significantly shorter and the anginal symptoms were significantly more frequent in the control group. In addition, the patients in the control group were more likely to have a severe clinical picture at presentation, with Killip class $>$ I. Systolic blood pressure (BP) was significantly higher in the control group, but heart rate did not differ between the 2 groups.

The patients in WS group were more likely to have $\mathrm{T}$ wave anomalies ( +/- biphasic or negative $\mathrm{T}$ waves) at presentation, while the patients in the control group were more likely to have ST segment depression.

TABLE 1. Baseline demographic, clinical and noninvasive data

\begin{tabular}{|c|c|c|c|}
\hline & $\begin{array}{c}\text { Group I - WS } \\
(n=64)\end{array}$ & $\begin{array}{c}\text { Group II - control } \\
(n=63)\end{array}$ & $\boldsymbol{P}$ \\
\hline Age, years & 62.38 & 62.38 & 0.997 \\
\hline Female gender, $\mathrm{n} \%$ & $20(31.7 \%)$ & $22(34.4 \%)$ & 0.85 \\
\hline Current smoker, n\% & $30(46.9 \%)$ & $22(34.9 \%)$ & 0.20 \\
\hline Arterial hypertension, $\mathrm{n} \%$ & $57(90.5 \%)$ & $56(87.5 \%)$ & 0.77 \\
\hline Dyslipidemia, $\mathrm{n} \%$ & $50(78.1 \%)$ & $52(82.5 \%)$ & 0.65 \\
\hline Diabetes mellitus, $\mathrm{n} \%$ & $31(48.4 \%)$ & $41(65.1 \%)$ & 0.07 \\
\hline History of angina pectoris, $n \%$ & $42(65.6 \%)$ & $35(55.6 \%)$ & 0.27 \\
\hline History of MI, n\% & $16(25.0 \%)$ & $22(34.9 \%)$ & 0.24 \\
\hline History of $\mathrm{PCl}, \mathrm{n} \%$ & $7(10.9 \%)$ & $13(20.6 \%)$ & 0.15 \\
\hline History of HF, n\% & $15(23.4 \%)$ & $23(36.5 \%)$ & 0.12 \\
\hline Other atherosclerotic manifestations, $\mathrm{n} \%$ & $8(12.5 \%)$ & $10(15.9 \%)$ & 0.62 \\
\hline Pretreatment with statins, $\mathrm{n} \%$ & $34(53.1 \%)$ & $24(38.1 \%)$ & 0.08 \\
\hline Pretreatment with antiplatelets, $\mathrm{n} \%$ & $16(25.0 \%)$ & $16(25.4 \%)$ & 0.75 \\
\hline Pretreatment with betablockers, $\mathrm{n} \%$ & $39(60.9 \%)$ & $33(52.4 \%)$ & 0.37 \\
\hline Onset-presentation time interval, h & 22.6 & 10.51 & 0.01 \\
\hline Angina pectoris at presentation, $\mathrm{n} \%$ & $53(82.8 \%)$ & $63(100 \%)$ & 0.001 \\
\hline Systolic BP at presentation, $\mathrm{mmHg}$ & 143.44 & 153.3 & 0.031 \\
\hline Heart rate at presentation, bpm & 82.66 & 86.9 & 0.253 \\
\hline Killip > I at presentation, $\mathrm{n} \%$ & $14(21.9 \%)$ & $24(38.1 \%)$ & 0.05 \\
\hline Biphasic/negative T waves, $\mathrm{n} \%$ & $38(59.4 \%)$ & $7(11.1 \%)$ & 0.001 \\
\hline ST segment depression, $\mathrm{n} \%$ & $4(6.3 \%)$ & $23(36.5 \%)$ & 0.001 \\
\hline hs-cTnl at presentation, $\mathrm{ng} / \mathrm{l}$ & 321.62 & 1567.39 & 0.053 \\
\hline Peak hs-cTnl, ng/l & 553.91 & 4029.12 & 0.002 \\
\hline NT-proBNP, pg/ml & 1204.43 & 1286.84 & 0.8 \\
\hline \multicolumn{3}{|l|}{ Mitral regurgitation } & \multirow{4}{*}{$\begin{array}{c}\text { global P } \\
0.565\end{array}$} \\
\hline mild, $\mathrm{n} \%$ & $41(64.1 \%)$ & $45(71.4 \%)$ & \\
\hline moderate, $\mathrm{n} \%$ & $14(21.9 \%)$ & $12(19 \%)$ & \\
\hline severe, $\mathrm{n} \%$ & $4(6.3 \%)$ & $2(3.2 \%)$ & \\
\hline LVEF,\% & 47.48 & 48.19 & 0.634 \\
\hline
\end{tabular}


High sensitive troponin I had significantly higher values in the control group, both at presentation and at peak, reflecting a larger area of myocardial necrosis. In contrast, the NT-proBNP value did not differ between the 2 groups, meaning that the severity of heart failure was similar.

The mean LVEF and the severity of mitral regurgitation (MR) were comparable between the 2 groups, although there were more patients with severe MR in WS group.

Risk assessment using GRACE 1.0 risk score was similar between the 2 groups, with mean values belonging to the low risk tertile, but with significantly different distribution considering that the majority of patients in WS group were at low risk. In-hospital predicted mortality using GRACE 1.0 was below $1 \%$ for both groups. The corresponding in-hospital and 6 months mortality risk estimation for both groups using GRACE 2.0 are shown in table 2.

Invasive characteristics and treatment indication are presented in table 3. Patients within WS group were more likely to have one vessel disease (46.9\% vs. $20.6 \%$ ), while patients within control group were more likely to have three vessel disease $(34.9 \%$ vs. $10.9 \%), p=0.02$. The culprit artery in WS group was mainly LAD $(66.1 \%$ vs. $44.3 \%, \mathrm{p}=0.002)$, followed by LM $(15.3 \%$ vs. $9.8 \%, \mathrm{p}=0.002)$. Surprisingly, although WS is clasically described in the anterior territory, there was a significantly higher proportion of RCA as culprit vessel in WS group (13.6\% vs. $6.5 \%)$.

Following coronary angiography, $65.62 \%$ of the patients in WS group and $53.96 \%$ of patients in control group were treated by ad-hoc PCI. The patients in the control group had a slightly higher proportion of surgical indication, but overall the treatment indication did not differ between the 2 groups (global $p=0.09$ ). For patients who benefited of PCI, we analysed the completeness of myocardial revascularization and we found no significant differences between the 2 groups $(88.1 \%$ in WS group vs. $81.1 \%$ in control group, $\mathrm{p}=0.53$ ).

Within 6 months of follow-up, patients in the control group had a significantly higher rate of cardiovascular rehospitalizations $(41.9 \%$ vs. $21.9 \%$, p $=0.016)$ (table 4).

TABLE 2. Risk assessment

\begin{tabular}{|c|c|c|c|}
\hline & $\begin{array}{l}\text { Group I - WS } \\
(n=64)\end{array}$ & $\begin{array}{l}\text { Group II - control } \\
\quad(n=63)\end{array}$ & $\boldsymbol{P}$ \\
\hline GRACE 1.0, points & 94.97 & 101.46 & 0.197 \\
\hline low risk ( $\leq 108$ points), $\mathrm{n} \%$ & $73.4 \%$ & $55.6 \%$ & \multirow{3}{*}{$\begin{array}{c}\text { global } p \\
0.042\end{array}$} \\
\hline moderate risk (109-140 points), $\mathrm{n} \%$ & $20.3 \%$ & $34.9 \%$ & \\
\hline high risk (>140 points), n\% & $6.3 \%$ & $9.5 \%$ & \\
\hline In-hospital GRACE 2.0,\% & 1.9234 & 2.5333 & 0.312 \\
\hline 6 months GRACE $2.0, \%$ & 4.3206 & 6.0278 & 0.079 \\
\hline
\end{tabular}

TABLE 3. Angiographic characteristics and treatment indication

\begin{tabular}{|c|c|c|c|}
\hline & $\begin{array}{l}\text { Group I - WS } \\
\quad(n=64)\end{array}$ & $\begin{array}{l}\text { Group II - control } \\
\qquad(n=63)\end{array}$ & $\boldsymbol{P}$ \\
\hline \multicolumn{4}{|l|}{ CAD inventory } \\
\hline one vessel, $\mathrm{n} \%$ & $30(46.9 \%)$ & $13(20.6 \%)$ & \multirow{4}{*}{ global P 0.02} \\
\hline two vessels, $n \%$ & $14(21.9 \%)$ & $15(23.8 \%)$ & \\
\hline three vessels, n\% & $7(10.9 \%)$ & $22(34.9 \%)$ & \\
\hline other, $\mathrm{n} \%$ & $10(15.6 \%)$ & $11(17.5 \%)$ & \\
\hline \multicolumn{4}{|l|}{ Culprit artery } \\
\hline LM, n\% & 9 (15.3\%) & 6 (9.8\%) & \multirow{4}{*}{$\begin{array}{c}\text { global P } \\
0.002\end{array}$} \\
\hline LAD, $n \%$ & $39(66.1 \%)$ & $27(44.3 \%)$ & \\
\hline LCX, n\% & $1(1.7 \%)$ & $17(27.9 \%)$ & \\
\hline $\mathrm{RCA}, \mathrm{n} \%$ & $8(13.6 \%)$ & $4(6.5 \%)$ & \\
\hline \multicolumn{4}{|l|}{ Treatment indication } \\
\hline no revascularization indication, $\mathrm{n} \%$ & $3(4.68 \%)$ & $2(3.17 \%)$ & \multirow{5}{*}{ global P 0.09} \\
\hline $\mathrm{PCl}, \mathrm{n} \%$ & $42(65.62 \%)$ & $34(53.96 \%)$ & \\
\hline CABG, $n \%$ & $10(15.6 \%)$ & $22(34.9 \%)$ & \\
\hline OMT, n\% & $7(10.93 \%)$ & $5(7.93 \%)$ & \\
\hline viability tests, $\mathrm{n} \%$ & $2(1.6 \%)$ & $0(0 \%)$ & \\
\hline
\end{tabular}


TABLE 4. Follow-up data

\begin{tabular}{|l|c|c|c|}
\hline & $\begin{array}{c}\text { Group I - WS } \\
\text { (n = 64) }\end{array}$ & $\begin{array}{c}\text { Group II - control } \\
\text { (n = 63) }\end{array}$ & $\boldsymbol{P}$ \\
\hline Cardiovascular rehospitalizations, $\mathrm{n} \%$ & $14(21.9 \%)$ & $26(41.9 \%)$ & 0.016 \\
\hline Ischaemic reccurences, $\mathrm{n} \%$ & $11(18.0 \%)$ & $14(22.2 \%)$ & 0.56 \\
\hline Subsequent revascularization, $\mathrm{n} \%$ & $9(14.8 \%)$ & $11(17.5 \%)$ & 0.31 \\
\hline Recurrent revascularization, $\mathrm{n} \%$ & $5(11.9 \%)$ & $6(17.6 \%)$ & 0.52 \\
\hline Repeat TVR, $\mathrm{n} \%$ & $2(4.9 \%)$ & $2(5.9 \%)$ & 1.00 \\
\hline Clinical bleeding, $\mathrm{n} \%$ & $7(10.9 \%)$ & $6(9.5 \%)$ & 1 \\
\hline Mortality, $\mathrm{n} \%$ & $4(6.3 \%)$ & $5(7.9 \%)$ & 0.74 \\
\hline
\end{tabular}

The rate of ischaemic recurrences was similar between the 2 groups. Other interventional endpoints, such as subsequent interventional revascularization (regardless the treatment at the beginning of the study), repeat interventional revascularization (in patients treated by PCI during the index hospitalization) and repeat target vessel revascularization (TVR) were comparable between the 2 groups. The proportion of clinically manifested bleedings was similar, but the patients in the control group had a significantly mean decrease of hemoglobin level from the initial value $(-0.78 \mathrm{~g} / \mathrm{dl}$ vs. $-0.35 \mathrm{~g} / \mathrm{dl}, \mathrm{p}=0.046)$. At the end of the 6 months follow-up period there was no significantly difference with respect to global mortality, $6.3 \%$ in WS group vs. $7.9 \%$ in the control group, $p$ $=0.74$ (figure 1 ).

\section{DISCUSSIONS}

In 1982 de Zwaan and Wellens described in patients with UA a particular ECG pattern consisting of symmetrically inverted or biphasic (+/-) $\mathrm{T}$ waves in leads V2 and V3, with unfavorable outcome if treated conservatively. Of the 26 patients with UA and the eponymous sign, 16 were managed pharmacologically and $75 \%$ of them developed extensive anterior MI. Only 10 patients underwent coronary angiography and all of them had a $\geq 90 \%$ stenosis of LAD [4].

The pathophysiological mechanism of Wellens sign is not yet fully understood. The most comprehensive explanation is the myocardial reperfusion lesion: a brief and transitory episode of severe my-

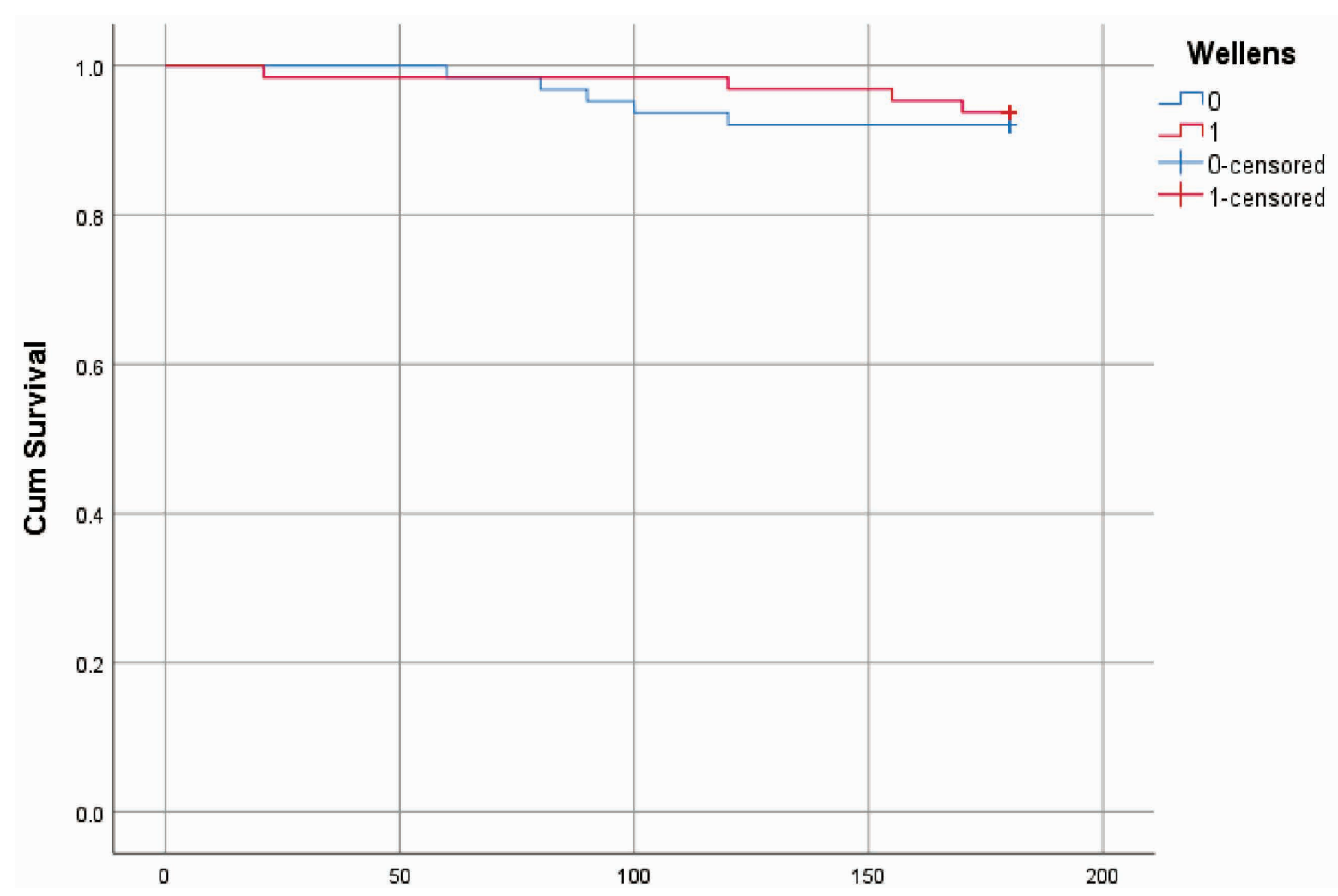

FIGURE 1. Kaplan-Meyer survival function for global mortality: red line - WS

group, blue line - control group 
ocardial ischaemia because of acute thrombotic occlusion of the culprit vessel due to plaque rupture, followed by spontaneous or pharmacologically facilitated reperfusion before myocardial necrosis development [6]. The ECG shows a minimal ST segment elevation while the vessel is occluded, then ST segment resolution once blood flow is restored and $\mathrm{T}$ wave anomalies after pain relief $[7,8]$. The ECG and angiographic characteristics of WS suggest an ,abortive” form of STEMI [9], proving the need for an updated definition of ECG signs of present or imminent coronary artery occlusion, especially LAD occlusion, as many patients may benefit from acute invasive treatment instead of conservative strategy [10].

Prevalence of Wellens sign in patients with UA in the first study of de Zwaan and Wellens was $18 \%$ (26 of 145 pts) [4]; in their later larger study the prevalence was $14 \%$ (180 of 1260 pts) [5]. It's worth mentioning that these studies took place before the wide use of cardiac biomarkers such as troponins. Thus, we can speculate that some of the patients in these early studies would have been diagnosed with NSTEMI if troponin dosing had been available. The prevalence and prognostic implications of Wellens sign in a contemporary cohort of patients with NSTEACS has not been yet clarified. Current review of literature revealed that it is plentiful of case reports of WS due to a variety of atherosclerothic or nonatherosclerothic causes (in-stent neoatherosclerosis [7], noncritical plaque coronary artery vasospasm $[8,11]$, coronary vasospasm due to illicit drugs use [12], spontaneous coronary artery dissection [13], myocardial bridging [14], coronary fistulae [15], stress cardiomyopathy [16]) or with alternative ECG and angiographic localization (inferior [17] or posterior [18] localization), but it is very scarce in studies.

Our study showed that the culprit artery in WS group was mainly LAD $(66.1 \%$ vs. $44.3 \%$, $\mathrm{p}=0.002)$, followed by LM $(15.3 \%$ vs. $9.8 \%$, $\mathrm{p}=0.002)$. These observations are consistent with the studies available so far. In a study, of the 24 patients with Wellens sign, two thirds had LAD culprit lesion $(66.7 \%$ vs. $19.6 \%, p<0.001)$, and the sensitivity of Wellens sign for LAD culprit prediction was $24.6 \%$ and the specificity was $96.2 \%$ [19]. Although WS is classically described in the anterior territory, we noticed a significantly higher proportion of RCA as culprit vessel in WS group $(13.6 \%$ vs. $6.5 \%, \mathrm{p}=0.002)$, unlike data in the literature. A recent study revealed that $20.8 \%$ of the patients with Wellens sign had RCA disease (stenosis $\geq 70 \%$ ), but none of them had RCA culprit artery [19]. Our study revealed that patients within WS group were more likely to have one vessel disease $(46.9 \%$ vs. $20.6 \%)$, while patients within control group were more likely to have three vessel disease $(34.9 \%$ vs. $10.9 \%), p=0.02$. In contrast, in a study patients with Wellens sign were more likely to have three vessel or LM disease $(4.2 \%$ vs. $20.4 \%, p=0.057)[19]$. We found that the overall treatment indication did not differ between the 2 groups (global $p=0.09$ ). The rate of interventional revascularization was similar across the 2 groups (65.62\% of the patients in WS group and $53.96 \%$ of patients in control group), as well as the rate complete revascularization $(88.1 \%$ in WS group vs. $81.1 \%$ in control group, $p=0.53$ ). In a similar study, the rate of revascularization procedures (PCI or CABG) was similar across the 2 groups and the rate of in-hospital PCI was comparable to our observations $(66.7 \%$ vs. $63.6 \%, \mathrm{p}=0.77)$, [19].

To our knowledge, this is the first prospective study with mid term follow up (6 months) that compared a consecutive cohort of patients with Wellens syndrome who underwent coronary angiography with an age and sex matched cohort of patients with NSTEACS. Within 6 months of follow-up, patients in the control group had a significantly higher rate of cardiovascular rehospitalizations $(41.9 \%$ vs. $21.9 \%, p=0.016)$, although the rate of ischaemic recurrences was similar between the 2 groups. Other interventional end-points, such as subsequent interventional revascularization, repeat interventional revascularization and repeat target vessel revascularization (TVR) were comparable between the 2 groups at 6 months follow-up. There was no significantly difference with respect to global mortality $(6.3 \%$ in WS group vs. $7.9 \%$ in the control group, $\mathrm{p}=0.74)$, although significantly more patients in WS group were in the low risk tertile $(73.4 \%$ vs. $55.6 \%, p=0.042)$ according to GRACE 1.0 risk score.

\section{Study limitations and strengths}

The most important limitation of the study is the relative small sample volume, mainly due to the rarity of WS. Another limitation is the monocentric nature of our study, with consequences on sample volume and local particularities related to the impact of operator and centre volume for PCI interventions on clinical outcomes.

The main strengths of this research are related to the study design. This is a prospective study, while all the studies published so far are retrospective. Also, our study is a longitudinal study, with a 
follow-up period of 6 months, while all studies available so far are related to the in-hospital period. Nonetheless, the control group is age and sex matched and this is reflected in the omogenicity of the basal characteristics of the patients.

\section{CONCLUSIONS}

Wellens sign is frequently overlooked in the emergency department. Prompt recognition of subtle ECG ischaemic changes in patients with chest pain or equivalents, such as Wellens sign, is

Conflict of interest: none declared

Financial support: none declared

\section{REFERENCES}

1. Ibanez B, James S, Agewall S, Antunes MJ, Bucciarelli-Ducci C, Bueno H, Caforio ALP, et al.; ESC Scientific Document Group. 2017 ESC Guidelines for the management of acute myocardial infarction in patients presenting with ST-segment elevation: The Task Force for the management of acute myocardial infarction in patients presenting with ST-segment elevation of the European Society of Cardiology (ESC). Eur Heart J. 2018 Jan 7;39(2):119-177.

2. Collet JP, Thiele $H$, Barbato $E$, Barthélémy $O$, Bauersachs J, Bhatt DL, Dendale P, Dorobantu M, et al.; ESC Scientific Document Group. 2020 ESC Guidelines for the management of acute coronary syndromes in patients presenting without persistent ST-segment elevation. Eur Heart J. 2021 Apr 7;42(14):1289-1367.

3. Asatryan B, Vaisnora L, Manavifar N. Electrocardiographic Diagnosis of Life-Threatening STEMI Equivalents: When Every Minute Counts. JACC: Case Reports. 2019;1(4):666-668.

4. de Zwaan C, Bär FW, Wellens HJ. Characteristic electrocardiographic pattern indicating a critical stenosis high in left anterior descending coronary artery in patients admitted because of impending myocardial infarction. Am Heart J. 1982 Apr;103(4 Pt 2):730-6.

5. de Zwaan C, Bär FW, Janssen JH, Cheriex EC, Dassen WR, Brugada P, Penn OC, Wellens HJ. Angiographic and clinical characteristics of patients with unstable angina showing an ECG pattern indicating critical narrowing of the proximal LAD coronary artery. Am Heart J. 1989 Mar;117(3):657-65.

6. Marzlin KM. Wellens Syndrome. AACN Adv Crit Care. 2018 Fall;29(3):360-364

7. Nisbet BC, Zlupko G. Repeat Wellens' syndrome: case report of critical proximal left anterior descending artery restenosis. J Emerg Med. 2010 Sep;39(3):305-8.

8. Abulaiti A, Aini R, Xu H, Song Z. A special case of Wellens' syndrome. J Cardiovasc Dis Res. 2013 Mar;4(1):51-4.

9. Stankovic I, Vlahovic-Stipac A, Ilic I, Putnikovic B, Neskovic AN. Aborted myocardial infarction in a patient with rapid progression of Wellens syndrome. J Emerg Med. 2012 Sep;43(3):e181-4. crucial as it reflects a large area of myocardium at risk and identifies a subgroup of patients in whom early invasive management can prevent natural evolution towards MI .

Risk assessment in WS based on risk scores validated for NSTEACS is frequently misleading. Although having clinical utility and being subject to continuous recalibration, clinical prediction rules like risk scores are imperfect instruments that underestimate the death risk in specific subgroups such as WS, in which clinical judgement should prevail.

10. Chioncel V, Avram A, Ion AC, Sinescu C. The de Winter T waves - an unusual presentation of left anterior descending artery occlusion. Romanian Journal of Cardiology. 2016;26(3):330-333.

11. Kukla P, Korpak-Wysocka R, Dragan J, Giszterowicz D, Dobrowolski W, Czamara M, Dudek D, Bryniarski L. Pseudo-Wellens syndrome in a patient with vasospastic angina. Kardiologia Polska. 2011; 69(1):79-81.

12. Co MLF, Das A, Okwuosa T. Pseudo-Wellens syndrome after heavy marijuana use. Cleve Clin J Med. 2017 Aug;84(8):590-591.

13. Migliore F, Zorzi A, Marra MP, Basso C, Corbetti F, De Lazzari M, Tarantini G, Buja P, Lacognata C, Thiene G, Corrado D, lliceto S. Myocardial edema underlies dynamic T-wave inversion (Wellens' ECG pattern) in patients with reversible left ventricular dysfunction. Heart Rhythm. 2011 Oct;8(10):1629-34.

14. Avram A, Chioncel V, Guberna S, Cuciureanu I, Brezeanu RC, Andrei $\mathrm{CL}$, Sinescu C. Myocardial bridging-an unusual cause of Wellens syndrome: A case report. Medicine (Baltimore). 2020 Oct 9;99(41):e22491.

15. Ibarrola M. Wellens' syndrome and finding of multiple coronary cameral fistulae: Is it time to discard this term? Ann Noninvasive Electrocardiol. 2020 Mar;25(2):e12693.

16. Singh M, Harlapur M, Acharya T, Wessel R, Bhullar A, Ambrose J. T wave and QT changes in Takotsubo cardiomyopathy versus Wellens T waves. J Am Coll Cardiol. 2016;67(13_S):524.

17. Chioncel V, Avram A, Sinescu C. A particular case of Wellens' Syndrome. Med Hypotheses. 2020 Nov;144:110013.

18. Driver BE, Shroff GR, Smith SW. Posterior reperfusion T-waves: Wellens' syndrome of the posterior wall. Emerg Med J. 2017 Feb;34(2):119-123.

19. Kobayashi A, Misumida N, Aoi S, Kanei Y. Prevalence and Clinical Implication of Wellens' Sign in Patients With Non-ST-Segment Elevation Myocardial Infarction. Cardiol Res. 2019 Jun; 10(3):135-141. 